Published by Cambridge University Press

This is an Open Access article, distributed under the terms of the Creative Commons

Attribution-NonCommercial-NoDerivatives licence (http://creativecommons.org/licenses/by-nc-nd/4.0/), which permits non-commercial re-use, distribution, and reproduction in any medium, provided the original work is unaltered and is properly cited. The written permission of Cambridge University Press must be obtained for commercial re-use or in order to create a derivative work.

doi:10.1017/S0022377820001300

\title{
Projections of H-mode access and edge pedestal in the SPARC tokamak
}

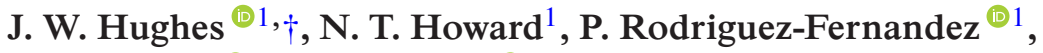 \\ A. J. Creely ${ }^{\circledR 2}$, A. Q. Kuang ${ }^{\circledR 1}$, P. B. Snyder ${ }^{3}$, T. M. Wilks ${ }^{1}$, \\ R. Sweeney ${ }^{\circledR 1}$ and M. Greenwald ${ }^{\circledR 1}$ \\ ${ }^{1}$ MIT Plasma Science and Fusion Center, Cambridge, MA 02139, USA \\ ${ }^{2}$ Commonwealth Fusion Systems, Cambridge, MA 02139, USA \\ ${ }^{3}$ General Atomics, San Diego, CA 92121, USA
}

(Received 12 June 2020; revised 16 September 2020; accepted 17 September 2020)

In order to inform core performance projections and divertor design, the baseline SPARC tokamak plasma discharge is evaluated for its expected $\mathrm{H}$-mode access, pedestal pressure and edge-localized mode (ELM) characteristics. A clear window for H-mode access is predicted for full field DT plasmas, with the available $25 \mathrm{MW}$ of design auxiliary power. Additional alpha heating is likely needed for $\mathrm{H}$-mode sustainment. Pressure pedestal predictions in the developed H-mode are surveyed using the EPED model. The projected SPARC pedestal would be limited dominantly by peeling modes and may achieve pressures in excess of $0.3 \mathrm{MPa}$ at a density of approximately $3 \times 10^{20} \mathrm{~m}^{-3}$. High pedestal pressure is partially enabled by strong equilibrium shaping, which has been increased as part of recent design iterations. Edge-localized modes (ELMs) with $>1 \mathrm{MJ}$ of energy are projected, and approaches for reducing the ELM size, and thus the peak energy fluence to divertor surfaces, are under consideration. The high pedestal predicted for SPARC provides ample margin to satisfy its high fusion gain $(Q)$ mission, so that even if ELM mitigation techniques result in a $2 \times$ reduction of the pedestal pressure, $Q>2$ is still predicted.

Key words: fusion plasma, plasma confinement

\section{Introduction}

The SPARC tokamak (Creely et al. 2020) is being designed to demonstrate significant fusion gain $(Q>2)$ for the first time, using a modestly sized device with high magnetic field enabled by recent advances in high-temperature superconductor technology. The basic parameters needed to achieve the performance goals of SPARC can be estimated using zero-dimensional (OD) analysis based on empirical confinement scalings, with increasing confidence provided by full 1.5D transport simulations, grounded in validated

† Email address for correspondence: jwhughes@psfc.mit.edu 
physics models (Rodriguez-Fernandez et al. 2020). Results of these efforts have a strong sensitivity to particular inputs. In the former case, the assumed H-factor is a significant lever on the $Q$ projected for SPARC. In the latter case, performance is varied in practice by the choice of pedestal boundary condition, i.e. plasma density and temperature at a normalized minor radius $r / a$ of perhaps $0.9-0.95$. Given these boundary conditions, the plasma $\mathrm{H}$-factor is determined self-consistently by evaluation of turbulent and neoclassical fluxes in the core. Owing to the stiff nature of the core transport in high-performance plasma conditions, the $\mathrm{H}$-factor is strongly dependent on the pedestal boundary condition (Rodriguez-Fernandez et al. 2020). The link between pedestal quality and H-factor is well established in existing experiments, for example in Hughes et al. (2011) and Leyland et al. (2015).

A high-quality pedestal also connects to some less-desirable features in high-energydensity tokamaks. Higher plasma pressure has been correlated with narrower scrape-off layer heat flux widths (Brunner et al. 2018; Faitsch et al. 2020; Silvagni et al. 2020), which could lead to increased challenges for divertor heat flux mitigation. In addition, high pedestal pressure is sometimes associated with large edge-localized modes (ELMs), transient phenomena that rapidly convert pedestal stored energy into bursts of energy and particles that must be borne by plasma-facing components (PFCs) in the main chamber and divertor (Leonard 2014).

The objective of this manuscript is to describe the methods used to make pedestal projections for SPARC, which can inform both the core performance projections and the divertor power handling requirements, as described in detail in separate papers (Kuang et al. 2020; Rodriguez-Fernandez et al. 2020). The methods described all have a basis in the extensive experience of the tokamak community. We focus on empirical scalings, semi-empirical scalings and model predictions that have received extensive validation on a number of existing experiments. SPARC represents a powerful opportunity to extend the validation of these models to new regimes of tokamak operation, allowing the closing of gaps in our physics projections to pilot plant concepts.

For reference, table 1 provides key input parameters needed to evaluate the various models described in the following. These are taken from the V2 design of SPARC (Creely et al. 2020). Section 2 describes the assumptions used to determine H-mode access on SPARC and the outlook for pedestal formation and sustainment. Section 3 motivates EPED as the constituent model for the pedestal and discusses predictions for SPARC device designs. Section 4 discusses the implications for ELM characteristics, including transient heat loading to be addressed in divertor design, as well as effects on fusion gain that would result from ELM mitigation techniques. Conclusions and directions for future work are given in $\S 5$.

\section{H-mode access}

\subsection{Empirical scaling for H-mode power threshold}

As on all tokamaks, access to a high pedestal on SPARC will require sufficient input power. In baseline high-performance operation this will enable a transition from L-mode to H-mode confinement and sustainment of the associated edge transport barrier. In the H-mode pedestal, a significant amount of mean flow shear is present to suppress turbulence, thus reducing cross-field transport and boosting confinement. Currently there is no first principles model that can deliver a general requirement for the critical flow shear, or translate flow shear into an input power requirement. Instead, the empirical approach has been pursued. L-H power threshold data from many machines have been gathered and regressed into scaling laws that can be projected, albeit imperfectly, to unbuilt 


$\begin{array}{lll}\text { Parameter } & \text { Symbol } & \text { Nominal value in full-field H-mode } \\ \text { On-axis toroidal field } & B_{0} & 12.2 \mathrm{~T} \\ \text { Major radius } & R_{0} & 1.85 \mathrm{~m} \\ \text { Minor radius } & a_{0} & 0.57 \\ \text { Plasma current } & I_{P} & 8.7 \mathrm{MA} \\ \text { Elongation at LCFS } & \kappa_{\text {sep }} & 1.97 \\ \text { Elongation at 99.5\% surface } & \kappa_{995} & 1.91 \\ \text { Triangularity at LCFS } & \delta_{\text {sep }} & 0.54 \\ \text { Triangularity at 99.5\% surface } & \delta_{995} & 0.49 \\ \text { Volume-averaged density } & \left\langle n_{e}\right\rangle & 3.1 \times 10^{20} \mathrm{~m}^{-3} \\ \text { Greenwald density limit } & n_{G} & 8.5 \times 10^{20} \mathrm{~m}^{-3} \\ \text { Maximum auxiliary ICRF power } & P_{\text {aux }} & 25 \mathrm{MW} \\ \text { Maximum fusion power } & P_{\text {fus }} & 140 \mathrm{MW} \\ \text { Global normalized beta } & \beta_{N} & 1.0 \\ \text { Effective charge } & Z_{\text {eff }} & 1.5 \\ \text { Average ion mass } & m_{i} & 2.5 \mathrm{amu}\end{array}$

TABLE 1. Full-field H-mode parameters for SPARC V2, useful as inputs to models found in the text.

devices (Ryter \& H Mode Database Working Group 1996; Takizuka \& ITPA H-mode Power Threshold Database Working Group 2004; Martin, Takizuka \& ITPA CDBM H-mode Threshold Database Working Group 2008). The prevailing example is a power law developed from a global multimachine database and adopted by the International Tokamak Physics Activity (ITPA) (Martin et al. 2008):

$$
P_{\mathrm{th}, \mathrm{ITPA}}=0.0488 n^{0.717} B^{0.803} S^{0.941}
$$

This scaling law was determined using actual $\mathrm{L}-\mathrm{H}$ power threshold data from several tokamaks: Alcator C-Mod, ASDEX Upgrade (AUG), DIII-D, JET, JFT-2M and JT-60 U. It represents the most widely accepted empirical model for power threshold, for deuterium tokamak plasmas, operated with the ion $B \times \nabla B$ drift directed toward a single magnetic null and at moderate to high normalized density. By way of illustration, figure 1 shows a comparison of (2.1) (blue solid curve) with experimental threshold power data from the Alcator C-Mod tokamak (Greenwald et al. 2014). Reasonable agreement is seen between the ITPA scaling law and the data over a typical range of density at which H-mode was accessed on the device: $1-2 \times 10^{20} \mathrm{~m}^{-3}$, with the caveat that the spread in the experimental data alone is easily a factor of two. With appropriate modifications, this scaling has been used exhaustively to project power requirements for ITER (ITER Organization, 2018), and can be applied to the design of SPARC.

One important modification is needed to account for the isotope scaling of $P_{\text {th }}$, because (2.1) was determined only for DD discharges. The preponderance of experience comparing $\mathrm{L}-\mathrm{H}$ power thresholds in $\mathrm{H}$ and $\mathrm{D}$ plasmas indicates an isotope scaling favourable to operation in DT. One quantitative study on JET determined a scaling of $P_{\text {th }}$ with $A^{-1}$ (Righi et al. 1999) and other tokamaks have demonstrated isotope scalings broadly consistent with this trend (Ryter et al. 2009, 2013; Behn et al. 2015; Yan et al. 2017). A linear reduction in power threshold with increasing isotope mass suggests that a 50:50 mix of DT would have its power threshold reduced by $20 \%$ relative to the projection from (2.1). 


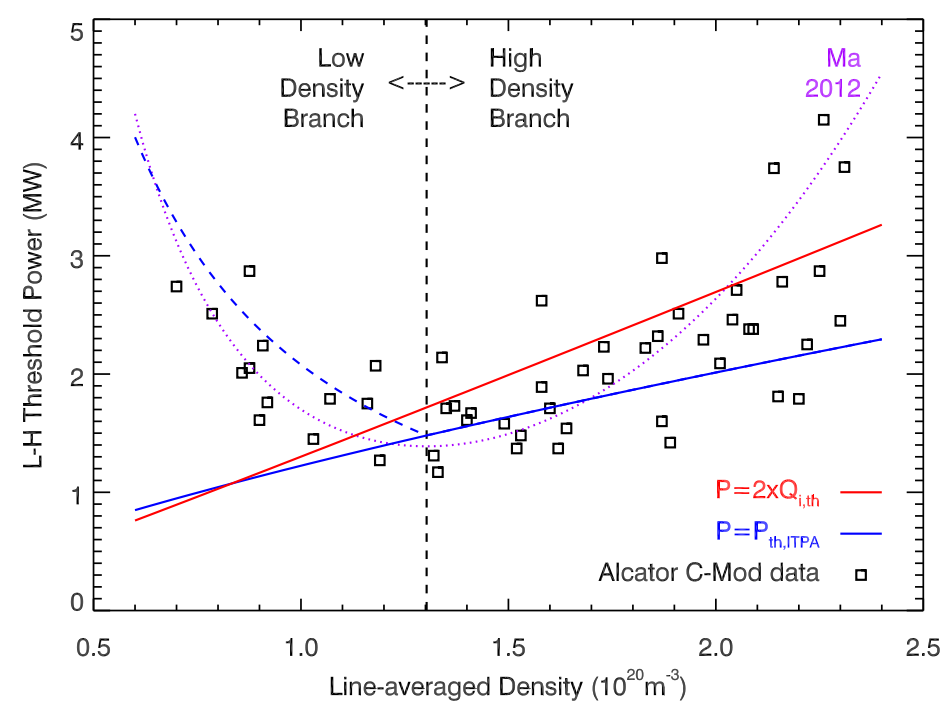

Figure 1. C-Mod $\left(B_{t}=5.4 \mathrm{~T}\right) \mathrm{L}-\mathrm{H}$ threshold power versus density, both data (squares) and various model curves. The dotted purple curve is from a previous fit to C-Mod data (Ma et al. 2012). The ITPA scaling law (Martin et al. 2008) is plotted as a solid blue curve. A scaling based on a critical ion heat flow model for the L-H threshold (Schmidtmayr et al. 2018) is shown in red, with $P_{\text {th }}=2 Q_{i}$,th. A novel low-density correction to the ITPA scaling law produces the dashed blue curve, which is important for $n<n_{\text {th, min }}$ as calculated according to (2.2) and illustrated by the vertical dashed line.

\subsection{Low-density limit for H-mode access}

Confidence in the ITPA scaling law is reduced at low density, because it was derived only with moderate- to high-density data from each device included. Neither (2.1) nor any other power law expressions for power threshold capture the tendency of a bifurcated dependence on density. Below a critical density, the power requirement takes on a negative dependence instead of positive, giving the curve a $U$-shape (Ryter et al. 2009; Gohil et al. 2011; Ma et al. 2012, Maggi et al. 2014). Although the ITPA scaling law agrees well with power threshold data from C-Mod taken in a standard range of operating density, figure 1 also clearly shows the non-monotonic density dependence of $P_{\text {th }}$ described in detail by Ma et al. (2012). When selected subsets of this type from within the international database are analysed, the density at the bottom of the $U$, i.e. where power is minimized, $n_{\mathrm{th} \text {, min }}$, is seen to have a negative dependence on $R$ and a positive dependence on $B_{T}$ (Martin et al. 2008). The positive trend of $n_{\mathrm{th} \text {,min }}$ with $B_{T}$ is also noted in experimental scans on individual devices (Ma et al. 2012, Maggi et al. 2014).

Several models have sought to capture and explain the existence of the power threshold reversal at low density (Fundamenski et al. 2012; Ryter et al. 2014; Bourdelle et al. 2015; Malkov et al. 2015). The strength of the thermal equilibration between ions and electrons in the core plasma may be a significant player in setting the transition between the low- and high-density branch of the power threshold. Following from the hypothesis that a critical ion heat flow through the plasma edge is necessary to sustain H-mode formation, Ryter et al. (2014) derived an expression for the density at minimum power threshold, i.e. at the bottom of the ' $U$ ':

$$
n_{\mathrm{th}, \min }=0.7 I_{P}^{0.34} B_{T}^{0.62} a^{-0.95}(R / a)^{0.4},
$$


with $n_{\mathrm{th}, \min }$ in $10^{19} \mathrm{~m}^{-3}, I_{P}$ in MA, $B_{T}$ in T and $R, a$ in $\mathrm{m}$. The expression agrees broadly with published values from C-Mod, AUG, JET and other devices (Ryter et al. 2014). When evaluated for the centroid of the data set in figure 1, equation (2.2) results in the vertical dashed line. This expression includes positive dependence on $B$ and negative dependence on machine size. For C-Mod and SPARC parameters, this formula suggests a transition to $e-i$ decoupling at low values of Greenwald fraction: $n / n_{G}<0.2$.

The Ryter model asserts a critical ion heat flow that is linear with density, a result that is found experimentally on both AUG and C-Mod. Combination of the data sets from the two machines (Schmidtmayr et al. 2018) allowed a combined regression

$$
Q_{i, \mathrm{th}}=0.0029 n^{1.05} B^{0.68} S^{0.93},
$$

with $Q_{i, \text { th }}$ in MW, $n$ in $10^{19} \mathrm{~m}^{-3}, B_{T}$ in $\mathrm{T}$ and $S$ in $\mathrm{m}^{2}$, which is very similar in character to the power law in (2.1) particularly considering the exponents on the field and surface area terms. An equivalent expression for total power threshold can be obtained by assuming a fraction of loss of power that is convected through the edge in the ion channel, $f_{i}=Q_{i} / P$. For typical C-Mod L-mode discharges in the high-density branch, we typically assume strong collisional coupling between electrons and ions and an ion power fraction of $f_{i}=0.5$. Power balance calculations from transport simulations are generally consistent with this assumption (Schmidtmayr et al. 2018), allowing us to take $P_{\text {th }}=2 \times Q_{i, \text { th }}$. Figure 1 shows that when this alternate expression is evaluated for C-Mod in the high-density branch, it loosely follows the experimental power threshold data, and is slightly more conservative than the ITPA expression.

Indications on AUG are that the critical ion heat flow required to initiate an $\mathrm{L}-\mathrm{H}$ transition approximately doubles when going from $\mathrm{D}$ to $\mathrm{H}$ discharges, consistent with the general isotope trend observed in total $P_{\mathrm{th}}$. (Ryter et al. 2013, 2014) This suggests that an isotope multiplier term $1 / A$ could be reasonably applied to the scaling in (2.3), as is often done for (2.1).

\subsection{Low-density limit for $\mathrm{H}$-mode access}

To explore H-mode power requirements for SPARC, we primarily use the ITPA scaling law in (2.1), modified accordingly for the assumed isotope mix. We anticipate this to be a useful estimate for $P_{\mathrm{th}}$, provided $n>n_{\mathrm{th}, \min }$. Below that critical density, an increase in the $P_{\text {th }}$ estimate is required. Following the model of Ryter et al. (2014), the details of the low-density branch (LDB) behaviour would be dependent on the mix of ion versus electron heating, and the details of transport. In the absence of these details, we can construct an ad hoc correction term to apply to the LDB, i.e.

$$
P_{\mathrm{th}, \mathrm{LDB}}=\left(n_{\mathrm{th}, \min } / n\right)^{2} P_{\mathrm{th}},
$$

where the density correction term is inspired by the $n^{2}$ dependence of the ion-electron energy exchange term, and is applied only for $n<n_{\mathrm{th}, \min }$. As shown in figure 1 , such a correction added to (2.1) does a respectable job capturing the experimental trend on C-Mod (dashed blue curve).

Sample projections of power thresholds to SPARC are shown in figure 2 and compared with expected values of available heating for the device, for both (a) DD plasmas and (b) DT plasmas. In each case, the ITPA scaling law from (2.1), modified via (2.2) and (2.4), is shown in blue. The $1 / A$ isotope correction is applied in figure $2(b)$, resulting in a $20 \%$ reduction of $P_{\mathrm{th}}$. SPARC will have $25 \mathrm{MW}$ of auxiliary wave heating power in the ion cyclotron range of frequencies (ICRF) (Lin, Wright \& Wukitch 2020). At full field, the minimum projected $P_{\text {th }}$ is close to this available power level, indicating that the 

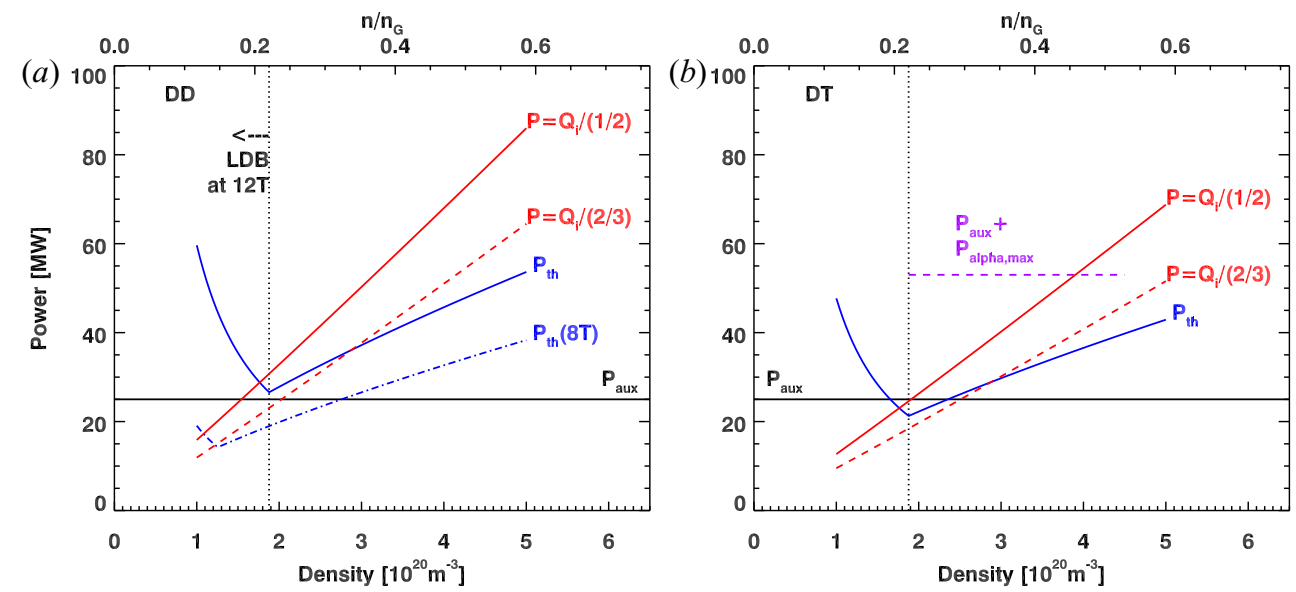

FIGURE 2. SPARC power threshold projections versus density in $(a)$ DD and $(b)$ DT operation. Blue curves represent the standard adopted for SPARC projections, a combination of expressions in (2.1), (2.2) and (2.4). Red curves represent projections using the expression for critical ion heat flow in (2.3) under assumptions that $Q_{i}=0.5 \times P$ (solid) and $Q_{i}=0.67 \times P$ (dashed). The curves in $(b)$ also factor in a $1 / A$ isotope scaling for power requirements. All curves are calculated using the baseline SPARC baseline parameters, except for the $8 \mathrm{~T} P_{\text {th }}$ curve in $(a)$. Horizontal lines indicate both the nominal auxiliary power available $(25 \mathrm{MW})$ as well as the maximum heating power available in DT when alpha power is included.

entry point to $\mathrm{H}$-mode will have to be near $n=n_{\mathrm{th}, \min }=1.9 \times 10^{20} \mathrm{~m}^{-3}$. The minimum $\mathrm{L}-\mathrm{H}$ threshold power for DT is projected to be $21 \mathrm{MW}$, providing a margin for H-mode access. Before tritium is introduced to the device, operations and scenario development will have to utilize deuterium-only discharges. The projection to the full field DD discharge indicates $P_{\text {th,min }}>25 \mathrm{MW}$. This makes H-mode access less likely for the DD scenario, although one cannot rule it out, given the level of scatter in actual power threshold observed in experiments, relative to scaling laws (see, for example, figure 1). An effective ICRF heating scheme exists for $2 / 3$ field operation at $8 \mathrm{~T}$, which does have a suitable margin for $\mathrm{L}-\mathrm{H}$ access. Therefore, the $8 \mathrm{~T}$ DD discharge is a likely candidate for initial $\mathrm{H}$-mode physics investigation on SPARC.

The ITPA scaling law in (2.1) remains the best accepted empirical scaling, owing to its multimachine nature and application on many subsequent devices. The projection of the LDB onset given by (2.2) has also shown an acceptable level of cross-machine validation. As they are broadly tested across tokamaks, these scalings have been chosen to inform the SPARC design, and inform the plasma operational contour analyses detailed in Creely et al. (2020) and Rodriguez-Fernandez et al. (2020). At the same time, it is of interest to track the projections obtained from the ion heat flow model. Power requirements based on (2.3) are shown in figure 2 for both DD and DT, with the latter adding a $1 / A$ isotope correction. Two values of ion heat flow fraction are assumed: $f_{i}=1 / 2$ (similar to the typical C-Mod assumption) and $f_{i}=2 / 3$, which may better approximate a strongly ion-heated scenario. The $\mathrm{L}-\mathrm{H}$ power requirements using these assumptions are similar to projections from the ITPA scaling law, for the nominal access condition of $n=n_{\mathrm{th} \text {, min }}$, but are significantly larger at higher density, owing to stronger dependence on $n$ seen in (2.3) versus (2.1). An important finding is that a high ratio of ion heat flow to total power can significantly lower the overall power requirement. This is seen in figure 2 by comparing the dashed red and solid blue curves at $n \sim 2 \times 10^{20} \mathrm{~m}^{-3}$. The ICRF heating technique on 
SPARC will use $120 \mathrm{MHz}$ RF minority heating on ${ }^{3} \mathrm{He}$ (Lin et al. 2020), which is projected to heat ions efficiently. Integrated simulations of the DT H-mode predict the majority of the edge heat flux to be in the ion channel (Rodriguez-Fernandez et al. 2020).

Inspection of figure 2 points to an additional consideration of $\mathrm{H}$-mode sustainment. There is no validated projection for $\mathrm{H}-\mathrm{L}$ back-transition power threshold in the same way that the ITPA scaling law provides for the $\mathrm{L}-\mathrm{H}$ power. However, once in $\mathrm{H}$-mode, maintaining power above $P_{\text {th }}$ (calculated at the increased density) generally grants confidence that $\mathrm{H}$-mode confinement will be sustained, although reductions in $\mathrm{H}$-factor and changes in ELM character may be observed at $P / P_{\text {th }}$ near unity (Ryter et al. 2008; Hughes et al. 2011; Knolker et al. 2018). On C-Mod, H-factor was shown to correlate with the ratio of net power flow through the pedestal $P_{\text {net }}$ to the calculated threshold power (Hughes et al. 2011). Although achieving this criterion becomes more challenging following the density increase associated with $\mathrm{H}$-mode formation, in DT the sum of auxiliary power and the maximum available alpha power in $\mathrm{H}$-mode provide headroom over the projected $P_{\text {th }}$, as seen in figure $2(b)$.

\subsection{Additional considerations for power threshold}

Confidence in the various power threshold projections is earned through validation on multiple machines, and activities continue within the community to refine power threshold projections, particularly within the ITPA. As updates from these activities emerge, they may be considered for revised SPARC projections. Meanwhile, we address a few considerations of SPARC device features that might potentially modify the $P_{\text {th }}$ projection from the standard ITPA expression.

First, although single-null operation of SPARC is possible, a double-null equilibrium is preferred for purposes of divertor heat load sharing and maintaining high elongation. $\mathrm{L}-\mathrm{H}$ $P_{\text {th }}$ scalings are well characterized only for single-null geometries with ion $B \times \nabla B$ drift toward the $\mathrm{X}$-point. We know from existing experimental work that balanced double-null operation does not obviously increase $P_{\text {th }}$ and can indeed reduce it. The reduction is significant in the case of low-aspect-ratio devices (Meyer et al. 2006, 2011) and more modest (perhaps $30 \%$ ) in the case of conventional-aspect-ratio tokamaks (Meyer et al. 2006). Thus, provided good control of magnetic balance, the $P_{\text {th }}$ projection we use for SPARC is conservative. If magnetic balance drifts too far in the direction of a single $\mathrm{X}$-point opposite the $B \times \nabla B$ direction, then significant increases in $\mathrm{L}-\mathrm{H}$ power can be expected.

Our projections are also not expected to increase due to the exclusive use of wave heating, as opposed to neutral beam injection (NBI), and the absence of driven rotation. The ITPA scaling law was fitted to a mix of beam-heated and wave-heated discharges across devices, including exclusively ICRF-heated cases on C-Mod (see again figure 1). The AUG findings discussed above point to a clear difference in threshold power for NBI versus electron cyclotron heated discharges, at low density, but this was attributed to the poor ion-electron equilibration in the LDB. Staying above $n_{\mathrm{th} \text {, min }}$, as well as providing a significant amount of ion heating with the ICRF, removes this as a concern on SPARC. Similarly, the lack of externally applied torque is not expected to raise the L-H power requirement. Scans of input torque on DIII-D have shown the zero crossing has substantially lower $P_{\text {th }}$ than the more typical case in which NBI power drives rotation in the co- $I_{P}$ direction (McKee et al. 2009), and this has been linked experimentally to reduced edge toroidal rotation, which facilitates the formation of a sheared edge radial electric field (Gohil et al. 2010). 
Magnetic field ripple, resulting from having discrete toroidal field coils, can affect plasma transport and influence rotation, and therefore is also a consideration for power thresholds. On JET, ripple at the outboard midplane separatrix was scanned up to $1.1 \%$. Although NBI losses increased at the higher ripple value, once these power losses are accounted for, power threshold was unaffected (Andrew et al. 2008). On DIII-D a local ripple of $3 \%$ was induced by a toroidally localized coil set, to simulate the effects of an ITER test blanket module. With the coil energized $P_{\text {th }}$ did not change outside uncertainties (Gohil et al. 2011). These relatively large levels of ripple exceed the target values for SPARC design. Ripple has been evaluated numerically for SPARC, mainly for the purpose of predicting ripple-induced fast ion losses (Scott et al. 2020). Considering both perfect alignment of coils and likely as-built displacements, the values of ripple remain below $0.5 \%$ at the nominal separatrix location, indicating low risk to $\mathrm{H}$-mode access.

The effects of divertor geometry and X-point location on the L-H threshold power can vary significantly on a given tokamak, introducing in some cases stronger than normal deviations from observed $P_{\text {th }}$, including significant reductions (Horton et al. 1999; Fukuda et al. 2000; Andrew et al. 2004, 2008; Gohil et al. 2011; Ma et al. 2012, Maggi et al. 2014). No unifying picture that explains the results on all devices has emerged, although the influence on divertor parameters on the upstream radial electric field in the scrape-off layer is a candidate mechanism that is actively under study (Chankin et al. 2017). The divertor effect remains one of the largest sources of variability in the projection of power threshold. The SPARC divertor is being designed to facilitate parallel heat flux mitigation via small inclination angle and strike point sweeping, and also to have a long outer leg to enable the creation of an X-point target (Kuang et al. 2020). This will create a significant variation on $\mathrm{X}$-point and divertor geometry across various scenarios and even within a single discharge, and may influence the entry into H-mode.

\section{Pedestal pressure predictions}

\subsection{Method for pedestal prediction}

Integrated transport modelling of SPARC has been effective at predicting the performance of SPARC and validating OD projections (Rodriguez-Fernandez et al. 2020), informing the device design. A vital ingredient for these simulations is a reliable prediction for the temperature and density boundary condition at the periphery. We obtain these from a model H-mode pedestal calculated using the EPED model (Snyder et al. 2011). EPED is the most widely validated predictive model available for the pedestal, having been tested on existing experiments spanning nearly two orders of magnitude in pedestal pressure (Groebner et al. 2013; Snyder et al. 2019). The agreement between EPED predictions and experimental values of pedestal height is typically within $20 \%$ across devices, for pedestals constrained by magnetohydrodynamic (MHD) stability. This includes the largest tokamak, JET, operating with both a carbon wall and an ITER-like wall of beryllium and tungsten (Beurskens et al. 2014). The cross-machine validation of EPED extends to high-field and high-density regimes on Alcator C-Mod (Walk et al. 2012; Tolman et al. 2018), including the highest-pressure pedestals ever achieved on a tokamak (Hughes et al. 2018). Extension of EPED to SPARC can be done with relatively high confidence. Though the absolute pressure on SPARC should exceed existing devices considerably, the pedestal beta and $\beta_{N}$ are within the range over which EPED has been validated.

EPED predicts the height and width of the pressure pedestal $\left(p_{\text {ped }}, \Delta_{\text {ped }}\right)$ as limited by criticality to kinetic ballooning mode and peeling-ballooning mode onset. The model equation for the pressure profile is a modified hyperbolic tangent function (Groebner et al. 2001), defined on coordinates of normalized poloidal flux $\psi$. Figure 3(a) gives an example 

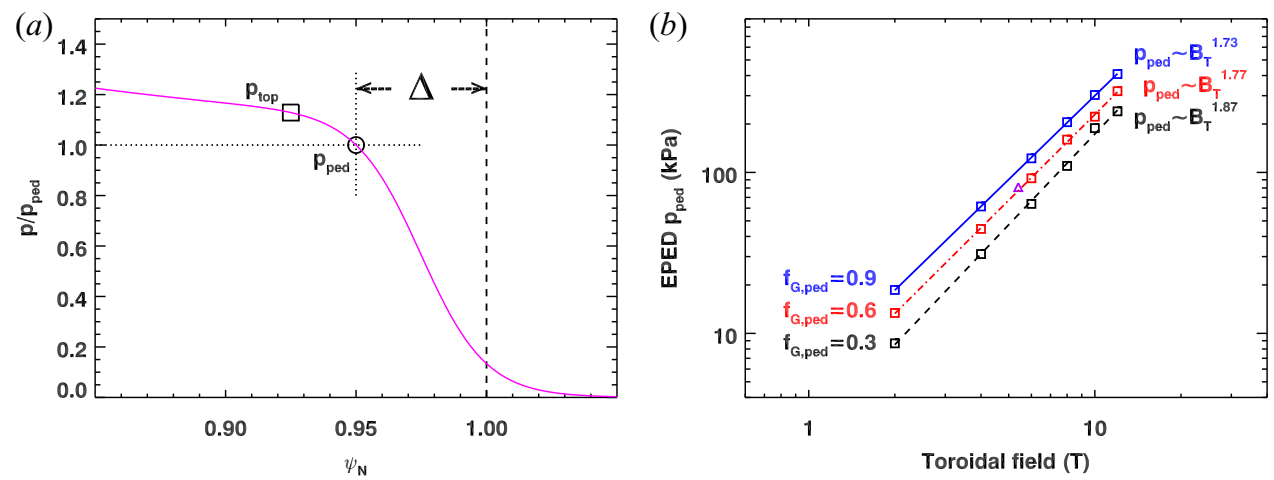

FIGURE 3. (a) Visualization of the width and height parameters of the modified tanh fit used in the EPED model, using an arbitrary pressure profile having width $\Delta=0.05$ in normalized poloidal flux. In this article, quantities subscripted 'ped' are taken one pedestal width inside the LCFS and those subscripted 'top' are taken 1.5 $\Delta$ inside the LCFS. (b) Scanning $B_{T}$ from $2 \mathrm{~T}$ to $12 \mathrm{~T}$ in a SPARC-sized tokamak, fixing edge $q$. The three scans are performed at indicated $f_{G \text {,ped }}$ values. The purple triangle indicates the highest experimental pedestal pressure reported previously, and the toroidal field at which the result was obtained (Hughes et al. 2018).

of this function. It exhibits a region of steep gradient with width $\Delta$, having a 'foot' at the location $\psi=1$ and a 'knee' at $\psi=1-\Delta$. The value of pressure at the knee defines $p_{\text {ped }}$. Similarly, $n_{\text {ped }}$ is defined as the density at the knee. Unless otherwise noted, the values of pedestal parameters in this article are taken at the knee. EPED also returns pressure values further up the profile at $\psi=1-1.5 \times \Delta$, which we refer to as $p_{\text {top }}$ and which get passed to the integrated transport model detailed in Rodriguez-Fernandez et al. (2020).

The pedestal pressure supplied by EPED is the maximum pedestal likely to be obtained, and is usually associated with a peeling-ballooning MHD stability boundary, which can be manifested experimentally either by Type I ELMs or in some cases by the formation of a quiescent $\mathrm{H}$-mode (QH-mode) pedestal with an edge harmonic oscillation (EHO) (Burrell et al. 2005; Solomon et al. 2015). A strength of the model is that its outputs are determined by a relatively simple set of scalar inputs. Most inputs are part of the design of a particular discharge: $B_{t}, I_{p}, R, a, \kappa, \delta, m_{i}$. Other inputs require additional prediction or estimation: $n_{\text {ped }}, \beta_{N, \text { global }}, Z_{\text {eff. }}$. Pedestal density is an example of an input with significant uncertainty, and which, as shown in the following, has a substantial effect on predicted pressure. To the extent pedestal density can be controlled, it can be considered an optimization parameter for pedestal and overall fusion performance.

A persistent finding from pedestal stability calculations, as embodied in EPED, is that low-collisionality H-modes with sufficiently strong shaping exhibit a positive $p_{\text {ped }}$ scaling with both toroidal and poloidal field (Snyder et al. 2015; Hughes et al. 2018). A consequence of this is that one may fix a tokamak plasma of a certain shape and safety factor, and obtain a much stronger than linear increase in $p_{\text {ped }}$ with $B_{T}$. Figure $3(b)$ shows an example of such scans, which begin with EPED pedestal predictions using a set of plasma parameters characteristic of a high-performance mid-size tokamak, similar in scale to SPARC: $B_{T}=2 \mathrm{~T}, I_{p}=0.9 \mathrm{MA}, R=1.67 \mathrm{~m}, a=0.58 \mathrm{~m}, \kappa=1.8, \delta=0.55$, $m_{i}=2, \beta_{N}=2.8, Z_{\text {eff }}=1.8$. Three values of input $n_{\text {ped }}$ in units of $10^{19} \mathrm{~m}^{-3}$ are chosen: 2.5, 5.0 and 7.5. From these three base cases, the value of $B_{T}$ is scanned to $12 \mathrm{~T}$, with $I_{p}$ and $n_{\text {ped }}$ increased in proportion so that the values of safety factor $q$ and pedestal Greenwald fraction $f_{G \text {,ped }}$ are held fixed. The remaining EPED inputs are held fixed. 
The strong dependence on toroidal field in all cases is shown by the fitted power law curves. Tripling the toroidal field and current allows this equilibrium to access pedestal pressure characteristic of the largest value achieved previously (Hughes et al. 2018) (purple triangle), and the full six-fold increase grants access to pedestal pressure in excess of $0.3 \mathrm{MPa}$.

The pressure pedestal prediction from EPED is quite sensitive to the input $n_{\text {ped }}$, as well as $Z_{\text {eff }}$, because these largely determine the collisionality regime, and collisionality impacts the self-consistent bootstrap current in the edge barrier. The favourable scaling with both $B_{P}$ and $B_{T}$ is enabled by maintaining low $v^{*}$, which results in a pedestal limited by low- $n$ peeling modes (i.e. modes driven primarily by the bootstrap current gradient in the edge barrier region). For higher $v^{*}$, EPED tends to predict a transition to a pedestal limited by higher- $n$ modes more ballooning in nature, which can decrease the attainable $p_{\text {ped }}$, a result that has been validated in experiments (Snyder et al. 2015; Hughes et al. 2018). For sufficiently strong plasma shaping, it is feasible to access a peeling-limited branch at higher density. This is referred to as super H-mode when it is associated with multivalued pedestal pressure predictions from EPED at a given density (Snyder et al. 2015; Snyder et al. 2019). SPARC design uses conservative physics choices and does not seek super H-mode solutions to meet its performance goals. For the purposes of SPARC pedestal prediction, we take only the lowest value of pressure predicted by EPED at a given density, noting that super $\mathrm{H}$-mode solutions could provide an avenue for further performance optimization if the parametric trajectory of the pedestal density can be controlled.

\subsection{EPED predictions informing SPARC device design}

Scanning the input $n_{\text {ped }}$ in EPED allows us to verify that SPARC remains on the peeling limited branch. An early operating point chosen for SPARC (V0) (Greenwald et al. 2018) was evaluated with EPED in order to identify the detailed density dependence of the pressure pedestal. The V0 parameters that differ from the V2 parameters in table 1 are as follows: $B_{T}=12 \mathrm{~T}, I_{p}=7.5 \mathrm{MA}, R=1.65 \mathrm{~m}, a=0.5 \mathrm{~m}, \kappa=1.8$ and $\delta=0.4$. V0 is smaller, is more weakly shaped and supports lower plasma current. Figure 4 illustrates the results of the scan, indicating predicted ( $a$ ) pedestal pressure, both in terms of $p_{\text {ped }}$ and $p_{\text {top }}$, and $(b)$ pedestal width. As seen in figure 4 , the predicted pedestal width and pressure were found to increase with $n_{\text {ped }}$ for the range of assumed operating densities, which spans about $2-4 \times 10^{20} \mathrm{~m}^{-3}$. This is a signature of peeling limited modes limiting the pedestal, and is very favourable to upward excursions in density, which do not result in a loss of performance. In contrast, operation at higher densities $\left(f_{G, \text { ped }}>0.55\right)$ would make the pedestal more ballooning limited, and lead to a $p_{\text {ped }}$ that decreases with increasing $n_{\text {ped }}$. For this and subsequent iterations, we have verified sufficient distance between the prospective operating density and the ballooning branch. This follows naturally because all iterations of SPARC design have aimed to be far from the Greenwald density limit (i.e. $\left.n / n_{G}<0.4\right)$.

Although elongation appears in confinement scaling laws, higher-order shaping parameters such as triangularity and squareness are notably absent. Nonetheless higher triangularity is shown in experiments to improve pedestal height and overall performance in H-mode (Osborne et al. 2000; Maggi et al. 2015), and this largely may be understood through the improvement in pedestal stability (Snyder et al. 2004). Starting from the modestly shaped case (delta $=0.4$ ) in figure 4 , we calculate the $p_{\text {ped }}$ change in an increasing scan of triangularity. The results shown in figure 5(a) indicate significant improvement with triangularity for $f_{G, \text { ped }}<0.4$. This pedestal enhancement motivated consideration of stronger shaping in subsequent SPARC design iterations (such as the current V2 iteration), as it would increase the maximum possible performance of the device. Although in this 

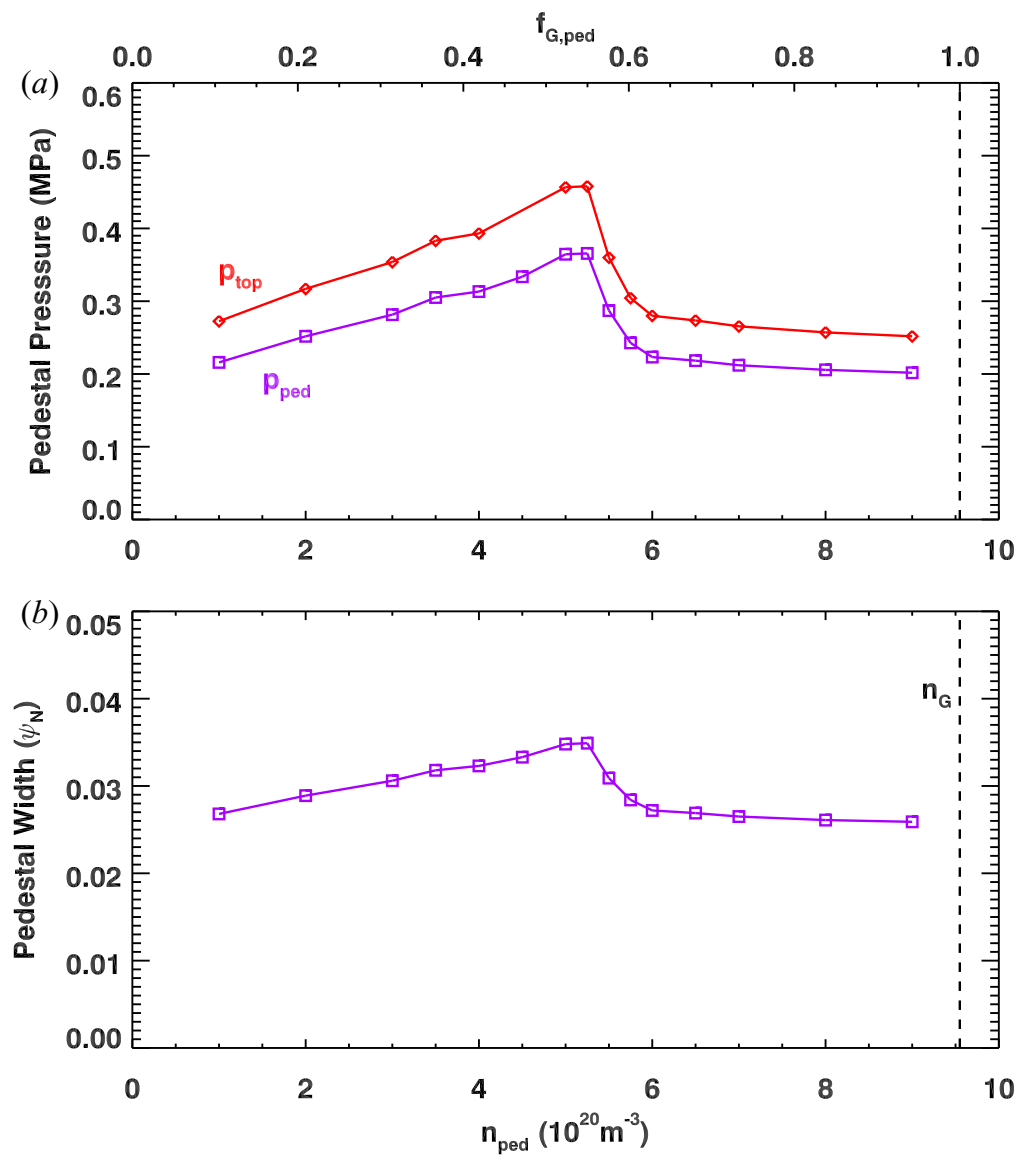

FIGURE 4. EPED density scan for SPARC V0 illustrating both peeling and ballooning limited branches: $(a) p_{\text {ped }}$ and $p_{\text {top }}$ as a function of assumed pedestal density (and pedestal Greenwald fraction); and $(b)$ pedestal width. The density dependence is familiar from simulation + analysis of existing devices.

particular scan the available operational space in the peeling branch is reduced from $f_{G \text {,ped }}<0.55$ to $f_{G \text {,ped }}<0.4$, a larger window for high-pressure operation is obtained by increasing elongation, as in figure 5(b), which results in increased edge $q$.

As the SPARC design parameters were iterated toward V2, predictions for pedestal height were embedded self-consistently into performance calculations (Rodriguez-Fernandez et al. 2020), and were thus able to reflect the equilibrium shape changes that occurred at each step. Realistic equilibria from TRANSP were used to generate the shaping inputs to EPED. Typical SPARC discharges under consideration are strongly elongated and have two poloidal field nulls, resulting in $\delta$ and $\kappa$ being significantly larger when evaluated on the separatrix, rather than on slightly interior flux surfaces inside the pedestal region. Given the increase in pressure pedestal with higher shaping shown previously, we chose a conservative approach and adopted the slightly lower values of $\delta$ and $\kappa$ that are calculated inside the pedestal region, at $\psi=0.995$ (see table 1). Requirements on divertor heat flux mitigation make strike point sweeping necessary, which introduces time-dependent triangularity values during a SPARC discharge (Kuang et al. 2020). The strike point sweep extrema are illustrated in 

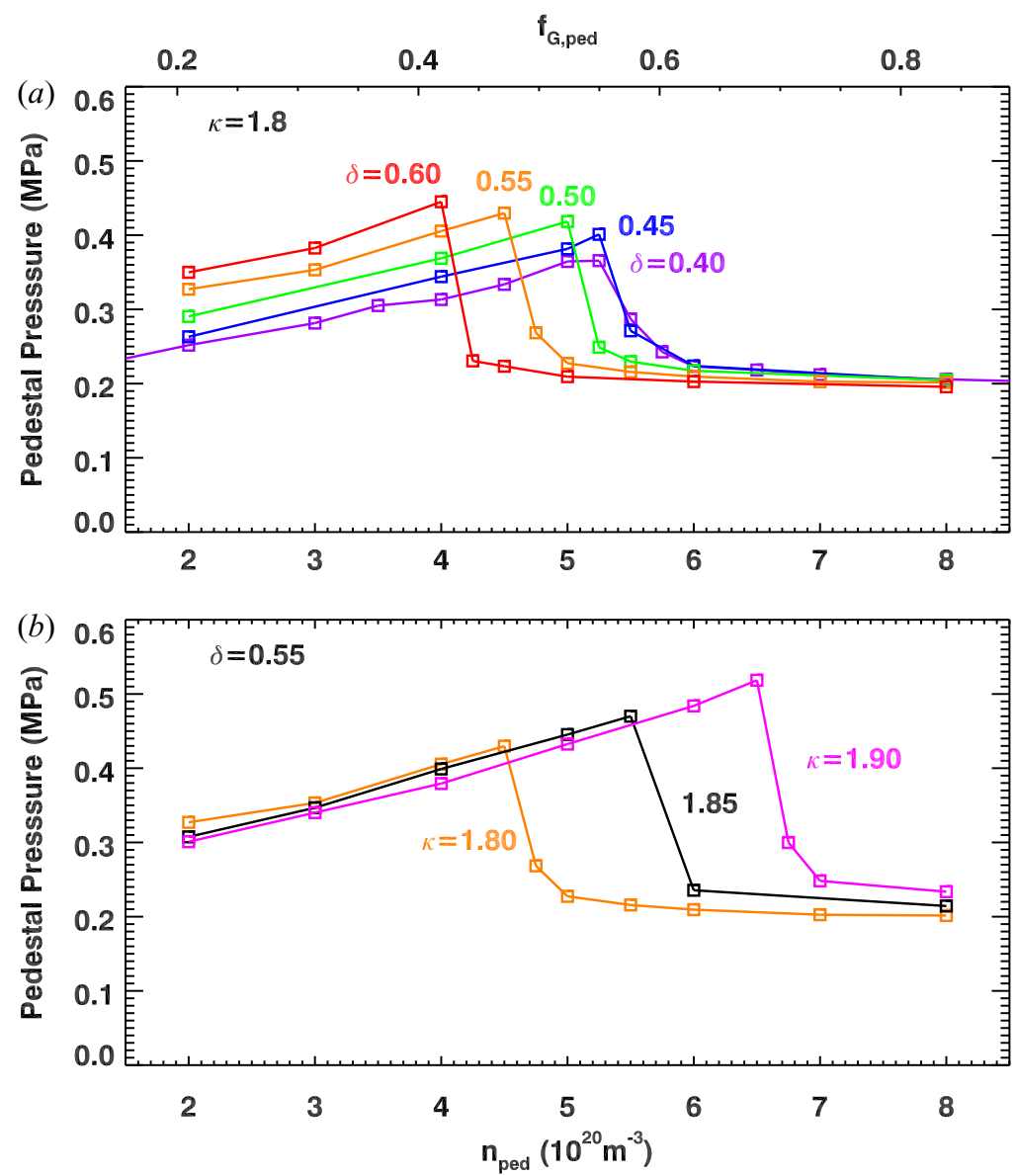

FIGURE 5. EPED predictions can directly probe shaping influence on SPARC performance. (a) The V0 case from figure 4 re-evaluated with progressively increasing triangularity (0.4-0.6). (b) The $\delta=0.55$ case re-evaluated with increasing elongation (1.8-1.9).

figure 6( $a$ ) with comparison with the V2 baseline. These sweeps alter the shape parameters from $\left[\delta_{995}=0.49, \kappa_{995}=1.91\right]$ to $\left[\delta_{995}=0.53, \kappa_{995}=1.88\right]$ for the inward swing and to $\left[\delta_{995}=0.44, \kappa_{995}=1.91\right]$ for the outward swing.

Using these values as input to EPED, the $p_{\text {ped }}$ predictions for V2 are calculated in figure $6(b)$. For the baseline (black squares) as well as the strike point extrema (red, blue), clear improvement is seen relative to the V0 design (purple), with higher obtainable pedestal pressure and a transition to the lower-performance ballooning-limited regime pushed to higher density (not shown). SPARC V2 remains on the peeling-limited branch for pedestal densities over a factor of two higher than the target value (vertical dashed line), indicating that modestly increasing the operating $\mathrm{H}$-mode density will not degrade pedestal performance. Note that these calculations assume up-down asymmetry of the equilibrium such that the upper and lower triangularities are equal. However, it is likely possible to design sweeps of the upper and lower divertor strike points which are out of phase, such that the average triangularity deviates less from that of the baseline, and the EPED predictions remain closer to the black curve in figure $6(b)$, throughout the sweep. 

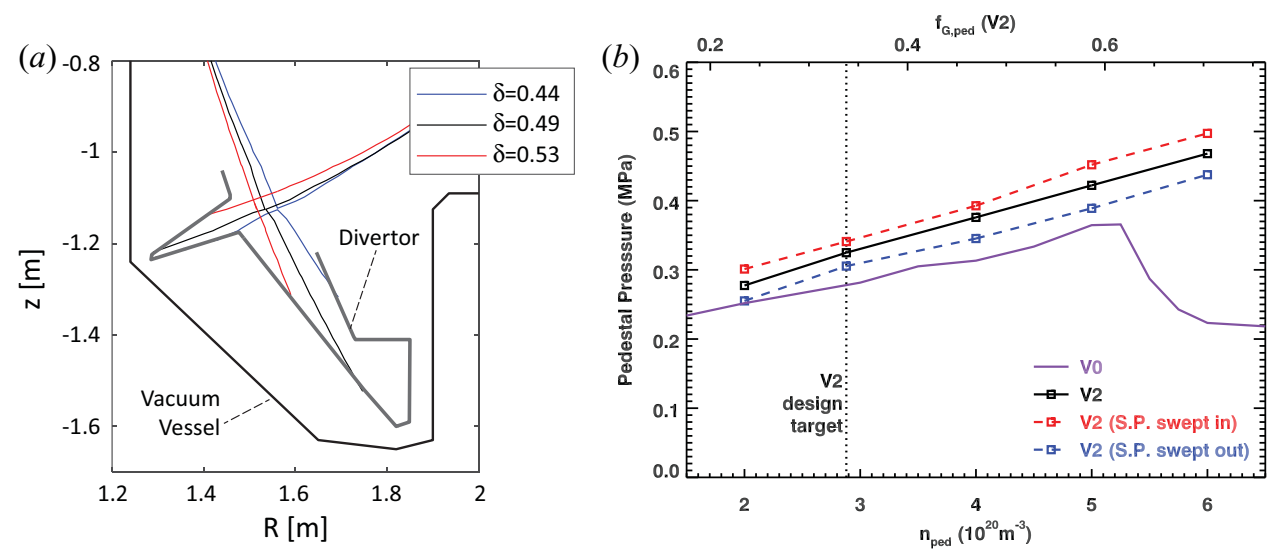

FIGURE 6. (a) Divertor region separatrix contours from SPARC V2 model equilibria: baseline (black) and with excursions from strike point sweeping (red, blue). (b) Pedestal predictions for the equilibria in $(a)$. The attainable pedestal performance significantly exceeds that of the V0 plasma (purple). The pedestal Greenwald fraction on the upper $x$-axis applies only to the V2 scans.

This may be desirable both for maintaining high pedestal pressure and for avoiding the onset of edge MHD.

\section{Expectations for edge transients}

\subsection{Unmitigated ELMs}

Tokamaks commonly exhibit periodic ELMs (Leonard 2014) in high-temperature H-modes. The typical ELM accompanying high performance is a Type I ELM, which manifests as a prompt drop in the plasma pressure at the top of the H-mode pedestal, and is accompanied by a burst of energy and particles onto the tokamak divertor, as well as onto main chamber surfaces. The pedestal regulation from ELMs would not directly limit SPARC performance, based on the pedestal projections in $\S 3$, which yield favourable overall confinement projections satisfying the SPARC mission of achieving $Q>2$ (Rodriguez-Fernandez et al. 2020). ELMs can be effective at removing impurities from the core plasma, however large ELMs create additional power and particle handling challenges for PFCs, and ELM size can correlate with pedestal pressure. We now turn to projecting the characteristics of natural ELMs, absent of external mitigation schemes, to guide PFC design, particularly components in the divertor.

First, we seek to quantify the size of an ELM, $\Delta W_{\mathrm{ELM}}$, which is equal to the ELM-induced drop in pedestal stored energy, $W_{\text {ped }}=3 / 2 \times p_{\text {ped }} \times V_{\text {plasma }}$. Here $W_{\text {ped }}$ is calculated using the EPED predictions in $\$ 3$ and shown in figure $7(a)$ along with the pedestal temperature. Multimachine studies conducted to inform ITER have concluded that the fractional ELM size $\Delta W_{\mathrm{ELM}} / W_{\text {ped }}$ correlates inversely with collisionality at the top of the pedestal $v_{\text {ped }}^{*}$ and this relationship is adopted to project unmitigated ELM size to ITER (Loarte et al. 2003, 2007). Figure 7(b) indicates the neoclassical collisionality calculated using pedestal parameters. From inspection of Loarte et al.'s (2007) figure 17, a range of projected $\Delta W_{\mathrm{ELM}} / W_{\text {ped }}$ values can be determined and used to compute a range of projected $\Delta W_{\text {ELM }}$ as a function of $n_{e \text {,ped. }}$. The minimum and maximum values are shown in figure 7(c), with the dashed verticals signifying points that have $P_{\text {th }}>P_{\text {aux }}+P_{\text {alpha, max }}$ and that are therefore likely inaccessible. At the SPARC design density, these assumptions give $1.4<\Delta W_{\mathrm{ELM}}[\mathrm{MJ}]<2.2$ with significant spread arising from the scatter in the 
(a)
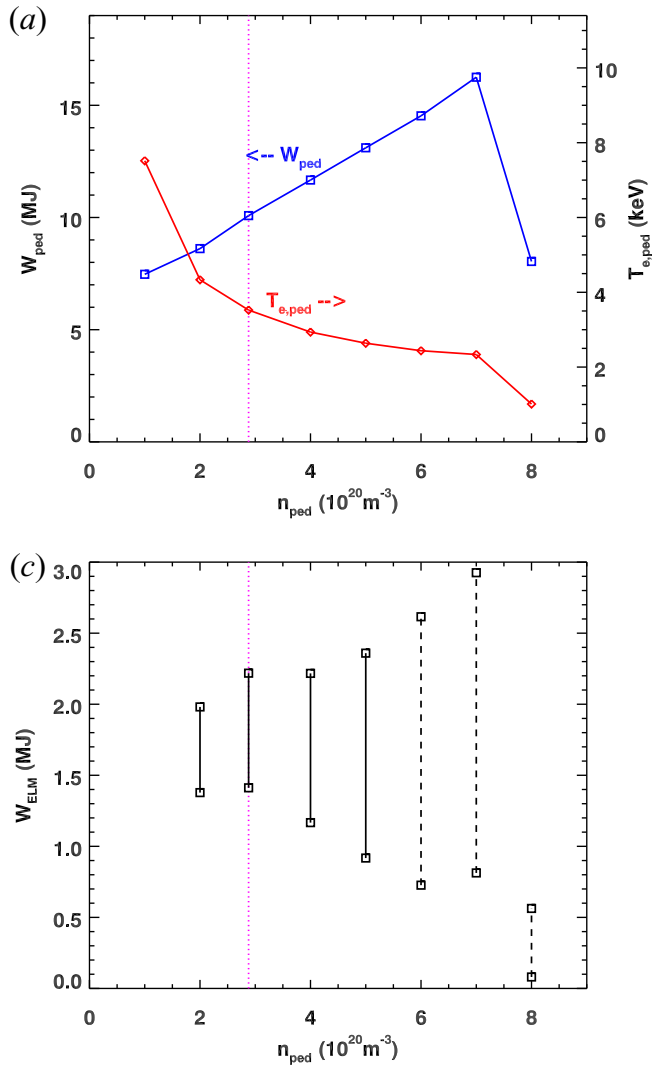

(b)

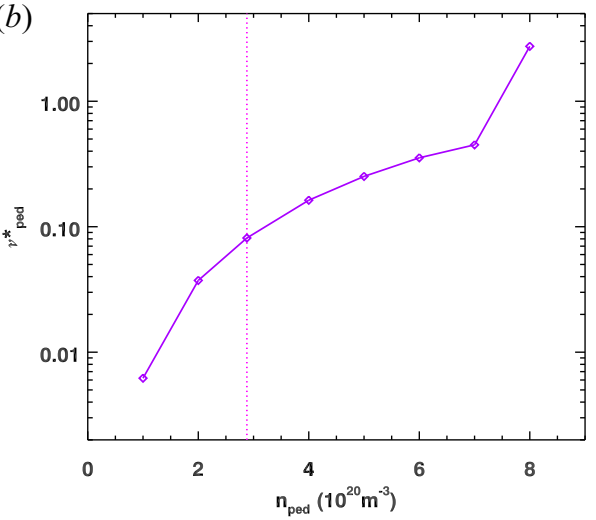

$(d)$

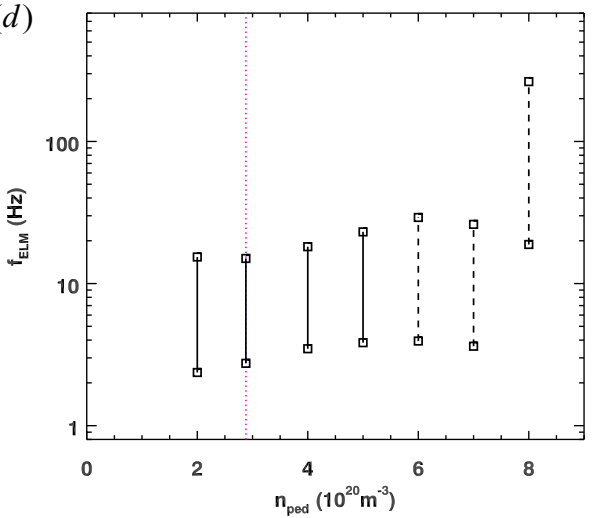

FIGURE 7. (a) Pedestal stored energy and pedestal temperature versus assumed pedestal density. (b) Pedestal neoclassical collisionality used to project $\Delta W_{\mathrm{ELM}} / W_{\text {ped. }}$ (c) Range in ELM energy content projected using multimachine data from Loarte et al. (2007). (d) Projected range in ELM frequency.

multimachine data (Loarte et al. 2007). Were the pedestal pressure at time of ELM to be less than the EPED value of $p_{\text {ped }}, v_{\text {ped }}^{*}$ would increase and the estimated range of $\Delta W_{\mathrm{ELM}}$ would fall accordingly.

Second, using the projections described previously, we can estimate a range of ELM frequency $f_{\text {ELM }}$, based on experimental findings that Type I ELMs tend to transport a fraction of total heating power (Hermann 2002), such that

$$
\Delta W_{\mathrm{ELM}} f_{\mathrm{ELM}}=C_{f} P_{\text {heat }},
$$

where $C_{f}$ ranges from 0.2 to 0.4 (Leonard et al. 1999). Figure $7(d)$ illustrates the range in $f_{\text {ELM }}$ for SPARC using the spread in $C_{f}$ and $\Delta W_{\text {ELM }}$ as inputs, and taking a realistic range for $P_{\text {heat }}$. At each density, the maximum value of $P_{\text {heat }}$ is taken to be $P_{\text {aux }}+P_{\text {alpha, max }}$, and the minimum value is taken as the lesser of $P_{\text {aux }}+P_{\text {alpha,max }}$ and $P_{\text {th. }}$. At the design density, we project a range of $2.7<f_{\text {ELM }}[\mathrm{Hz}]<15.0$. Increased ELM frequency would be expected if the pressure pedestal was below the EPED prediction, and the ELM energy was proportionally lower.

Megajoule-class ELMs will introduce a significant regular perturbation on the pedestal boundary condition. A periodic drop in the pedestal, and rebuild, have not to date been included in time-dependent simulations of SPARC performance, and represent an 
opportunity for further development. Another need is to relate these pedestal energy losses to transient heat loads on divertor surfaces. Here we adopt a semi-empirical approach rooted in multimachine database analysis and use a scaling developed for the peak energy fluence $\epsilon_{\| \text {,peak }}$, which is just the time integral of the instantaneous parallel heat flux associated with an ELM (Eich et al. 2017). A database of AUG, JET and MAST ELM energy fluence data is well represented by

$$
\epsilon_{\|, \text {peak }}=C 6 \pi p_{e} R_{\text {geo }} q_{\text {edge }},
$$

where $p_{e}$ is the pedestal electron pressure $(\mathrm{Pa}), R_{\mathrm{geo}}$ is the geometric major radius $(\mathrm{m})$ and $q_{\text {edge }}$ is the edge cylindrical safety factor. Here $C$ is a factor that ranges from 1 to 3 , where $C=3$ captures the upper bound of the fluences in the data set and $C=1$ captures the lower bound. Using the projections described previously and approximating $p_{e}=p_{\text {ped }} / 2$, we obtain a peak energy fluence of $11-32 \mathrm{MJ} \mathrm{m}^{-2}$, which is similar in magnitude to projections for ITER (Eich et al. 2017). Based on the database used to generate (4.2), ELM loads are statistically larger for larger values of $W_{\mathrm{ELM}} / W_{\text {plasma }}$, i.e. $\sim 10 \%$ rather than $\sim 2 \%$. Unmitigated ELMs on SPARC are likely to be at the high end of this range. Using a set of assumptions for the spatial and temporal distribution of this energy, a range of surface heat flux factor can be calculated and compared with the limits of candidate divertor materials (Kuang et al. 2020). To increase margin with respect to the ELM loading limits imposed by divertor PFCs, ELM mitigation schemes require consideration in SPARC.

\subsection{Implications for performance with ELM mitigation}

Mitigation or even full suppression of ELMs is feasible in tokamaks, both through extrinsic ELM control strategies (Lang et al. 2013) and operation in intrinsically ELM-suppressed regimes (Lang et al. 2013; Maingi 2014). Mitigation of ELMs typically involves the reduction of ELM magnitude, and often an increase in ELM repetition rate, using auxiliary means. One potential mitigation scheme for SPARC would involve pacing the ELMs with pellet injection (Lang et al. 2004; Romanelli, Kamendje on behalf of JET-EFDA Contributors 2009). One necessary requirement for this technique would be the regular injection of pellets at frequency $f_{\text {pellet }}>f_{\text {ELM }}$ (i.e. in the $20 \mathrm{~Hz}$ range or larger). Work is also ongoing to evaluate the feasibility of applying resonant magnetic perturbations (RMPs), which have had success on other devices in mitigating ELMs (Evans et al. 2004; Liang et al. 2007; Suttrop et al. 2011; Jeon et al. 2012; Kirk et al. 2013; Sun et al. 2016). Full suppression of ELMs is also possible via application of RMPs, although the window for suppression is narrower than that for mitigation. Error field correction coils currently being designed for SPARC are planned to be capable also of introducing $n=3$ RMPs, at an amplitude sufficient to satisfy the commonly used vacuum island overlap width criterion for complete ELM suppression (Fenstermacher et al. 2008; Evans et al. 2013). The ELM energy loading study (Eich et al. 2017) used to generate (4.2) included analysis of mitigated regimes and determined that this expression for ELM energy fluence was equally valid, i.e. the ELM load on the divertor continued to scale with the pedestal pressure under mitigation. There are also several demonstrated high-performance regimes with intrinsic ELM suppression. Examples include EDA H-mode (Hubbard et al. 2001; Hughes et al. 2013), I-mode (Whyte et al. 2010; Hughes et al. 2013; Walk et al. 2014), QH-mode with EHOs (Burrell et al. 2005; Solomon et al. 2015; Wilks et al. 2018) and wide pedestal QH-mode (Burrell et al. 2016). Future work will explore the feasibility of access to these regimes. As in the case of ELM mitigation, a reduction in pedestal pressure is often (though not always) observed with intrinsic ELM suppression. 


$\begin{array}{lccc}T_{e, \text { ped }} / T_{e, \text { ped }}^{(\text {EPED })} & 1.0 & 0.67 & 0.5 \\ \text { Maximum } \epsilon_{\|, \text {peak }} & 32 & 22 & 16 \\ \text { Modelled } Q & 9.0 & 5.1 & 3.0 \\ \text { Modelled } \mathrm{H}_{98} & 1.0 & 0.87 & 0.75\end{array}$

TABLE 2. Reduced ELM energy fluence and core performance from downgrading the EPED pedestal prediction by one-third and one-half.

Because techniques we attempt to reduce the ELM loads in SPARC will likely result in a reduction in pedestal pressure below the unmitigated ELMy H-mode limit (i.e. what is predicted using EPED), it is important to account for this 'penalty' on the pedestal height when projecting the performance of a particular scenario. This pedestal reduction has been investigated for SPARC using the previously discussed integrated modelling tools, in which the temperature pedestal is tuned below the default prediction from EPED, while leaving the target density the same (Rodriguez-Fernandez et al. 2020). The reduction of maximum ELM energy fluence is shown in table 2, alongside the resulting reduction in fusion gain and model $\mathrm{H}$-factor. Because SPARC is designed with significant performance margin, even a stringent ELM mitigation or suppression technique that halves the pedestal pressure will still allow the device to meet its performance mission.

\section{Conclusions and future directions}

The discussion presented here has shown that a combination of empirical scalings and modelling can be used to constrain performance projections for the SPARC tokamak, with a focus on the V2 design. In keeping with a conservative design philosophy, we have chosen models for H-mode access, pedestal height and ELM size that have been validated across multiple devices, and largely conform to community standards for projection to ITER. Accessing high-performance discharges on SPARC will test all these models in a new kind of fusion environment, on a device that uses very high magnetic fields at modest size, combines low collisionality with high absolute density and derives a significant amount of its heating power from fusion alpha particles. Insights gained from validating these models in the SPARC tokamak has the potential to significantly improve our confidence in model projections to future devices, including fusion pilot plant concepts.

$\mathrm{H}$-mode access is computed using a standard ITPA scaling law in (2.1), with a modification that significantly increases the power requirement at $n<n_{\mathrm{th} \text {, min }}$. In the baseline 12.2 T DT discharge, we find $\mathrm{H}$-mode to be accessible with some margin using available auxiliary power. Projections indicate a range in density of $1.7-2.3 \times 10^{20} \mathrm{~m}^{-3}$ in L-mode at which H-mode triggering is likely with $25 \mathrm{MW}$ of coupled ICRF. H-mode access in DD discharges may require operation at reduced field, e.g. 8 T. Following the $\mathrm{L}-\mathrm{H}$ transition, increased core particle content will raise the value of $P_{\mathrm{th}}$, and we thus expect $\mathrm{H}$-mode sustainment to require significant alpha heating. An alternate empirical scaling, based in the physics of a critical ion heat flow, is also assessed in SPARC, and suggests that $P_{\text {th }}$ for the $\mathrm{L}-\mathrm{H}$ transition could be lower than anticipated, given enough ion heating. Because the ion heat scaling in (2.3) is only partially validated across devices, and because it is not readily translated to an absolute power requirement, it is not used to guide the device design. We are actively pursuing refinements to SPARC power threshold projections, based on emerging experimental and modelling developments in the community. 
The EPED model generates a set of pressure pedestal predictions given SPARC operational parameters and assumptions for the pedestal density. These predictions support the high field approach by demonstrating a stronger than linear scaling of $p_{\text {ped }}$ with $B_{T}$ (at fixed $q_{95}$ ) in a medium sized, moderately shaped tokamak. Pedestal performance optimization is straightforward with EPED as design parameters are changed, and the positive influence of shaping $(\delta, \kappa)$ is clearly demonstrated. This influence propagates through integrated core performance modelling, and is internalized in design iterations. Partly because of this, as SPARC V0 evolved to V2, stronger shaping parameters were realized. Throughout the evolution of the design, we found that at targeted Greenwald fractions, the $\mathrm{H}$-mode pedestal remained on the peeling-limited branch of operation found at low $v_{\text {ped }}^{*}$. Thus, pedestal performance is very favourable, and $T_{\text {ped }}$ is stable to upward excursions of density.

Some uncertainties remain in the pedestal predictions for SPARC. One physics gap is the ability to predict the structure of the density pedestal. Currently $n_{\text {ped }}$ is an input to be scanned, because further development is needed before we can make believable predictions for the pedestal density. These will depend on accurate models for particle source rate from both edge fuelling and pellet injection, as well as on better characterization of diffusive and convective (including inward) particle transport in the pedestal. Improved models for the density pedestal could allow us to relate the pedestal density to the separatrix density, providing a better means of coupling with divertor/SOL models. We note that significant edge fuelling has been a regular requirement on many devices for managing core impurity accumulation and stationarity (Kallenbach et al. 2009; Nunes et al. 2013; Beurskens et al. 2014; Nunes on behalf of the JET Contributors 2016). Pedestal pressure degradation and confinement loss is often observed in such cases and has been linked to an outwardly shifted density pedestal (Hughes et al. 2011; Dunne et al. 2017; Stefanikova et al. 2018; Frassinetti et al. 2019). In H-modes limited by peeling-ballooning modes, the degradation may be explained by reduced stabilization of higher $n$ peeling-ballooning modes, which is a consequence of reduced pedestal bootstrap current and magnetic shear. As noted by Frassinetti et al. (2019) for low collisionality and high triangularity, as the dominant instabilities in the pedestal shift to the lower $n$ peeling branch, this effect is expected to become less important. The design point of SPARC is such that it is solidly on the peeling branch and, therefore, we expect radial shifts of the density pedestal to be benign for MHD stability. Future work will test this expectation with full stability calculations using variable density pedestal structure.

Other factors that could cause departures from EPED predictions include fundamental changes to the stability of turbulent modes, which may result in pedestals not limited by peeling-ballooning modes, and which have differing limits on pedestal gradient or width. Such mechanisms are likely already present in intrinsically ELM-suppressed regimes studied on existing devices (Maingi 2014) and may even play a strong role in a subset of JET ELMy H-modes with high gas puff, for which Frassinetti et al. (2019) shows experimental $p_{\text {ped }}$ approximately $30 \%$ below EPED predictions. In addition, mitigation schemes such as ELM pacing and RMP application will most likely reduce the pedestal. Given the requirement for ELM mitigation and additional uncertainties in the density profile discussed previously, we routinely take the EPED prediction to be the maximum achievable pressure pedestal. The performance margin built into SPARC allows it to meet its $Q>2$ goal even with up to a $2 \times$ reduction in $p_{\text {ped }}$ from the EPED prediction. Future work will include development of empirical models for the pedestals not limited by peeling-ballooning modes, and also access conditions to those regimes in terms of power and density requirements. 


\section{Acknowledgements}

This work was funded by Commonwealth Fusion Systems under RPP005. Alcator C-Mod data were acquired and analysed prior to 2016 under DoE Award DE-FC02-99ER54512. Part of the data analysis was performed using the OMFIT integrated modelling framework (Meneghini et al. 2015) Development and testing of EPED is supported by DE-FG02-95ER54309 and DE-FC02-06ER54873. We thank the SPARC Physics Basis group for helpful discussions, in particular, J. Rice and C. Paz-Soldan.

Editor William Dorland thanks the referees for their advice in evaluating this article.

\section{Declaration of interests}

The authors report no conflict of interest.

\section{REFERENCES}

Andrew, Y., Hawkes, N. C., O’Mullane, M. G., Sartori, R., de BaAr, M., Coffey, I., Guenther, K., Jenkins, I., Korotkov, A., Lomas, P., et al. 2004 JET divertor geometry and plasma shape effects on the L-H transition threshold. Plasma Phys. Control. Fusion 46, A87-A93.

Andrew, Y., Biewer, T. M., Crombe, K., Keeling, D., De la luna, E., Giroud, C., Hawkes, N. C., Kempenaars, M., Korotkov, A., Meigs, A., et al. 2008 H-mode access on JET and implications for ITER. Plasma Phys. Control. Fusion 50, 124053.

Behn, R., Labit, B., Karpushov, A., Martin, Y., Porte, L. \& TCV Team 2015 Threshold power for the transition into H-mode for H, D, and He plasmas in TCV. Plasma Phys. Control. Fusion 57, 025007 .

Beurskens, M. N. A., Frassinetti, L., Challis, C., Giroud, C., Saarelma, S., Alper, B., Angioni, C., Bilkova, P., Bourdelle, C., Brezinsek, S., et al. 2014 Global and pedestal confinement in JET with a Be/W metallic wall. Nucl. Fusion 54, 043001.

Bourdelle, C., Chôné, L., Fedorczak, N., Garbet, X., Beyer, P., Citrin, J., Delabie, E., Dif-Pradalier, G., Fuhr, G., Loarte, A., et al. 2015 L to H mode transition: parametric dependencies of the temperature threshold. Nucl. Fusion 55, 073015.

Brunner, D., LaBombard, B., Kuang, A. Q. \& Terry, J. L. 2018 High-resolution heat flux width measurements at reactor-level magnetic fields and observation of a unified width scaling across confinement regimes in the Alcator C-Mod tokamak. Nucl. Fusion 58, 094002.

Burrell, K. H., West, W. P., Doyle, E. J., Austin, M. E., Casper, T. A., Gohil, P., Greenfield, C. M., Groebner, R. J., Hyatt, A. W., Jayakumar, R. J., et al. 2005 Advances in understanding quiescent H-mode plasmas in DIII-D. Phys. Plasmas 12, 056121.

Burrell, K. H., Barada, K., Chen, X., Garofalo, A. M., Groebner, R. J., Muscatello, C. M., Osborne, T. H., Petty, C. C., Rhodes, T. L., Snyder, P. B., et al. 2016 Discovery of stationary operation of quiescent $\mathrm{H}$-mode plasmas with net-zero neutral beam injection torque and high energy confinement on DIII-D. Phys. Plasmas 23, 056103.

Chankin, A. V., Delabie, E., Corrigan, G., Maggi, C. F. \& Meyer, H. 2017 Possible influence of near SOL plasma on the H-mode power threshold. Nucl. Mater. Energy 12, 273-277.

Creely, A. J., Greenwald, M. J., Ballinger, S. B., Brunner, D., Canik, J., Doody, J., FÜlÖP, T., GARnier, D. T., GRANETZ, R., GRAY, T. K., et al. 2020 Overview of the SPARC tokamak. J. Plasma Phys. 86. doi:10.1017/S0022377820001257.

Dunne, M. G., Potzel, S., Reimold, F., Wischmeier, M., Wolfrum, E., Frassinetti, L., Beurskens, M., Bilkova, P., Cavedon, M., Fischer, R., et al. 2017 The role of the density profile in the ASDEX Upgrade pedestal structure. Plasma Phys. Control. Fusion 59, 014017.

EICH, T., et al. 2017 ELM divertor peak energy fluence scaling to ITER with data from JET, MAST and ASDEX upgrade. Nucl. Mater. Energy 12, 84-90.

Evans, T. E., Moyer, R. A., Thomas, P. R., Watkins, J. G., Osborne, T. H., Boedo, J. A., Doyle, E. J., Fenstermacher, M. E., Finken, K. H., Groebner, R. J., et al. 2004 
Suppression of large edge-localized modes in high-confinement DIII-D plasmas with a stochastic magnetic boundary. Phys. Rev. Lett. 92 (23), 235003.

Evans, T. E., Orlov, D. M., Wingen, A., Wu, W., Loarte, A., Caspar, T. A., Schmitz, O., SAibene, G., Schaffer, M. J. \& DALY, E. 2013 3D vacuum magnetic field modelling of the ITER ELM control coil during standard operating scenarios. Nucl. Fusion 53, 093029.

FAitsch, M., EICH, T., Sieglin, B. \& JET CONTRIBUtors 2020 Correlation between near scrape-off layer power fall-off length and confinement properties in JET operated with carbon and ITER-like wall. Plasma Phys. Control. Fusion 62, 085004.

Fenstermacher, M. E., Evans, T. E., Osborne, T. H., Schaffer, M. J., Aldan, M. P., deGrassie, J. S., Gohil, P., Joseph, I., Moyer, R. A., Snyder, P. B., et al. 2008 Effect of island overlap on edge localized mode suppression by resonant magnetic perturbations in DIII-D. Phys. Plasmas 15, 056122.

Frassinetti, L., Dunne, M. G., Sheikh, U., SaArelma, S., Roach, C. M., Stefanikova, E., Maggi, C., Horvath, L., Pamela, S., DE la Luna, E., et al. 2019 Role of the pedestal position on the pedestal performance in AUG, JET-ILW and TCV and implications for ITER. Nucl. Fusion 59, 076038.

Fukuda, T., Takizuka, T., Tsuchiya, K., Kamada, Y. \& Asakura, N. 2000 Reduction of L-H transition threshold power under the W-shaped pumped divertor geometry in JT-60 U. Plasma Phys. Control. Fusion 42, A289-A297.

Fundamenski, W., Militello, F., Moulton, D. \& McDonald, D. C. 2012 A new model of the L-H transition in tokamaks. Nucl. Fusion 52, 062003.

Gohil, P., Evans, T. E., Fenstermacher, M. E., Ferron, J. R., Osborne, T. H., Park, J. M., Schmitz, O., Scovolle, J. T. \& UnterberG, E. A. 2011 L-H transition studies on DIII-D to determine H-mode access for operational scenarios in ITER. Nucl. Fusion 51, 103020.

Gohil, P., Jernigan, T. C., Osborne, T. H., Scoville, J. T. \& Strait, E. J. 2010 The torque dependence of the H-mode power threshold in hydrogen, deuterium and helium plasmas in DIII-D. Nucl. Fusion 50, 064011.

Greenwald, M., Bader, A., Baek, S., Bakhtiari, M., Barnard, H., Beck, W., Bergerson, W., Bespamyatnov, I., Bonoli, P., Brower, D., et al. 201420 years of research on the Alcator C-Mod tokamak. Phys. Plasmas 21, 110501.

Greenwald, M., Whyte, D., Bonoli, P., Hartwig, Z., Irby, J., LaBombard, B., Marmar, E., Minervini, J., TAKAYASU, M., Terry, J., et al. 2018 The high-field path to practical fusion energy. MIT Plasma Science and Fusion Center Research Report No. PSFC/RR-18-2.

Groebner, R. J., Baker, D. R., Burrell, K. H., Carlstrom, T. N., Ferron, J. R., Gohil, P., LaO, L. L., Osborne, T. H., Thomas, D. M., West, W. P., et al. 2001 Progress in quantifying the edge physics of the H mode regime in DIII-D. Nucl. Fusion 41, 1789-1802.

Groebner, R. J., Chang, C. S., Hughes, J. W., Maingi, R., Snyder, P. B., Xu, X. Q., Boedo, J. A., Boyle, D. P., CAllen, J. D., CANiK, J. M., et al. 2013 Improved understanding of physics processes in pedestal structure, leading to improved predictive capability for ITER. Nucl. Fusion 53, 093024.

Hermann, A. 2002 Overview on stationary and transient divertor heat loads. Plasma Phys. Control. Fusion 44, 883-903.

Horton, L., Vlases, G. C., Andrew, P., Bhatnagar, V. P., Chankin, A. V., Clement, S., Conway, G. D., Davies, S. J., De HaAs, J. C. M., EhrenberG, J. K., et al. 1999 Studies in JET divertors of varied geometry. I: non-seeded plasma operation. Nucl. Fusion 39 (1), 1.

Hubbard, A. E., Boivin, R. L., Granetz, R. S., Greenwald, M., Hughes, J. W., Hutchinson, I. H., Irby, J., LaBombard, B., Lin, Y., Marmar, E. S., et al. 2001 Pedestal profiles and fluctuations in C-Mod enhanced D-alpha H-modes. Phys. Plasmas 8 (5), 2033-2040.

Hughes, J. W., Loarte, A., Reinke, M. L., Terry, J. L., Brunner, D., Greenwald, M., Hubbard, A. E., LaBombard, B., Lipschultz, B., MA, Y., et al. 2011 Power requirements for superior H-mode confinement on Alcator C-Mod: experiments in support of ITER. Nucl. Fusion 51, 083007.

Hughes, J. W., Snyder, P. B., Walk, J. R., Davis, E. M., Diallo, A., LaBombard, B., Baek, S. G., Churchill, R. M., Greenwald, M., Groebner, R. J., et al. 2013 Pedestal structure 
and stability in H-mode and I-mode: a comparative study on Alcator C-Mod. Nucl. Fusion 53, 043016.

Hughes, J. W., Snyder, P. B., Reinke, M. L., LaBombard, B., Mordijck, S., Scott, S., Tolman, E., BAeK, S. G., Golfinopoulos, T., GRAnetZ, R. S., et al. 2018 Access to pedestal pressure relevant to burning plasmas on the high magnetic field tokamak Alcator C-Mod. Nucl. Fusion 58, 112003.

ITER ORGANIZATION 2018 ITER research plan within the staged approach. ITER Technical Report No. ITR-18-003.

Jeon, Y. M., Park, J.-K., Yoon, S. W., Ko, W. H., Lee, S. G., Lee, K. D., Yun, G. S., Nam, Y. U., KIM, W. C., KWAK, JONG-GU., et al. 2012 Suppression of edge localized modes in high-confinement KSTAR plasmas by nonaxisymmetric magnetic perturbations. Phys. Rev. Lett. 109, 035004.

Kallenbach, A., Dux, R., Mayer, M., Neu, R., Pütterich, T., Bobkov, V., Fuchs, J. C., Eich, T., Giannone, L., Gruber, O., et al. 2009 Non-boronized compared with boronized operation of ASDEX upgrade with full-tungsten plasma facing components. Nucl Fusion 49, 045007.

Kirk, A., Chapman, I. T., Liu, Yueqiang, Cahyna, P., Denner, P., Fishrool, G., Ham, C. J., HARRison, J. R., LiAng, Yunfeng, NARdon, E., et al. 2013 Understanding edge-localized mode mitigation by resonant magnetic perturbations on MAST. Nucl. Fusion 53, 043007.

Knolker, M., Bortolon, A., Canal, G. P., Evans, T. E., Zohm, H., Abrams, T., Buttery, R. J., Davis, E. M., Groebner, R. J., Hollmann, E., et al. 2018 Investigation of the role of pedestal pressure and collisionality on type-I ELM divertor heat loads in DIII-D. Nucl. Fusion 58, 096023

Kuang, A., Ballinger, S., Brunner, D., Canik, J., Creely, A. J., Gray, T., Greenwald, M., HugheS, J. W., IRBY, J., LABOMBARD, B., et al. 2020 Divertor heat flux challenge and mitigation in SPARC. J. Plasma Phys. 86. doi:10.1017/S0022377820001117.

Lang, P. T., Conway, G. D., Eich, T., Fattorini, L., Gruber, O., GÜnter, S., Horton, L. D., Kalvin, S., Kallenbach, A., KaUfmann, M., et al. 2004 ELM pace making and mitigation by pellet injection in ASDEX upgrade. Nucl. Fusion 44, 665-677.

Lang, P. T., Loarte, A., Saibene, G., Baylor, L. R., Becoulet, M., Cavinato, M., Clement-Lorenzo, S., Daly, E., Evans, T. E., Fenstermacher, M. E., et al. 2013 ELM control strategies and tools: status and potential for ITER. Nucl. Fusion 53, 043004.

LeOnard, A. W. 2014 Edge-localized-modes in tokamaks. Phys. Plasmas 21, 090501.

Leonard, A. W., Herrmann, A., Itami, K., Lingertat, J., Loarte, A., Osborne, T. H., Suttrop, W., THE ITER Divertor Modeling AND DATABASE ExPERT Group \& THE ITER Divertor PHySICS EXPERT GROUP 1999 The impact of ELMs on the ITER divertor. J. Nucl. Mater. 266-269, 109-117.

Leyland, M. J., Beurskens, M. N. A, Frassinetti, L., Giroud, C., SaArelma, S., Snyder, P. B., Flanagan, J., Jachmich, S., KempenaArs, M., Lomas, P., et al. 2015 The H-mode pedestal structure and its role on confinement in JET with a carbon and metal wall. Nucl. Fusion 55, 013019.

Liang, Y., Koslowski, H. R., Thomas, P. R., Nardon, E., Alper, B., Andrew, P., Andrew, Y., ARnouX, G., BARANOV, Y., BÉCOUlet, M., et al. 2007 Active control of type-I edge-localized modes with $n=1$ perturbation fields in the JET tokamak. Phys. Rev. Lett. 98, 265004.

Lin, Y., Wright, J. C. \& WUKITCH, S. J. 2020 Physics basis for the ICRF system of the SPARC tokamak. J. Plasma Phys. 86. doi:10.1017/S0022377820001269.

Loarte, A., Saibene, G., Sartori, R., Campbell, D., Becoulet, M., Horton, L., Eich, T., Herrmann, A., Matthews, G., Asakura, N., et al. 2003 Characteristics of type I ELM energy and particle losses in existing devices and their extrapolation to ITER. Plasma Phys. Control. Fusion $45,1549-1569$.

Loarte, A., Lipschultz, B., Kukushikin, A. S., Matthews, G. F., Stangeby, P. C., Asakura, N., Counsell, G. F., Federici, G., Kallenbach, A., Krieger, K., et al. 2007 Chapter 4: power and particle control. Nucl. Fusion 47, S203-S263. 
Ma, Y., Hughes, J. W., Hubbard, A. E., Labombard, B., Churchill, R. M., Golfinopoulos, T., TsujiI, N. \& MARMAR, E. S. 2012 Scaling of H-mode threshold power and L-H edge conditions with favourable ion grad-B drift in Alcator C-Mod tokamak. Nucl. Fusion 52, 023010.

Ma, Y., Hughes, J. W., Hubbard, A. E., LABombard, B. \& Terry, J. 2012 H-mode power threshold reduction in a slot-divertor configuration on the Alcator C-Mod tokamak. Plasma Phys. Control. Fusion 54, 082002.

Maggi, C. F., Delabie, E., Biewer, T. M., Groth, M., Hawkes, N. C., Lehnen, M., De La Luna, E., McCormick, K., ReuX, C., Rimini, F., et al. 2014 L-H power threshold studies in JET with Be/W and C wall. Nucl. Fusion 54, 023007.

Maggi, C. F., Saarelma, S., Casson, F. J., Challis, C., de la luna, E., Frassinetti, L., Giroud, C., Joffrin, E., Simpson, J., Beurskens, M., et al. 2015 Pedestal confinement and stability in JET-ILW ELMy H-modes. Nucl. Fusion 55, 113031.

MAINGI, R. 2014 Enhanced confinement scenarios without large edge localized modes in tokamaks: control, performance, and extrapolability issues for ITER. Nucl. Fusion 54, 114016.

Malkov, M. A., Diamond, P. H., Miki, K., Rice, J. E. \& Tynan, G. R. 2015 Linking the micro and macro: L-H transition dynamics and threshold physics. Phys. Plasmas 22, 032506.

Martin, Y. R., TAKizuka, T. \& ITPA CDBM H-MOde Threshold Database Working Group 2008 Power requirement for accessing the H-mode in ITER. J. Phys. Conf. Ser. 123, 012033.

McKee, G. R., Gohil, P., Schlossberg, D. J., Boedo, J. A., Burrell, K. H., DeGrassie, J. S., Groebner, R. J., Moyer, R. A., Petty, C. C., Rhodes, T. L., et al. 2009 Dependence of the L- to H-mode power threshold on toroidal rotation and the link to edge turbulence dynamics. Nucl. Fusion 49, 115016.

Meneghini, O., Smith, S. P., Lao, L. L., Izacard, O., Ren, Q., Park, J. M., Candy, J., WANG, Z., LUNA, C. J., Izzo, V. A., et al. 2015 Integrated modeling applications for tokamak experiments with OMFIT. Nucl. Fusion 55, 083008.

Meyer, H., Carolan, P. G., Conway, G. D., Cunningham, G., Horton, L. D., Kirk, A., Maingi, R., Ryter, F., SAArelma, S., Schirmer, J., et al. $2006 \mathrm{H}$-mode physics of near double null plasmas in MAST and its applications to other tokamaks. Nucl. Fusion 46, 64-72.

Meyer, H., De Bock, M. F. M., Conway, N. J., Freethy, S. J., Gibson, K., Hiratsuka, J., Kirk, A., Michael, C. A., Morgan, T., SCAnnell, R., et al. 2011 L-H transition and pedestal studies on MAST. Nucl. Fusion 51, 113011.

Nunes, I., Lomas, P. J., McDonald, D. C., Saibene, G., Sartori, R., Voitsekhovitch, I., Beurskens, M., Arnoux, G., Bовос, A., Еich, T., et al. 2013 Confinement and edge studies towards low $\rho^{*}$ and $v^{*}$ at JET. Nucl. Fusion 53, 073020.

NUNES, I. on behalf of the JET Contributors 2016 Plasma confinement at JET. Nucl. Fusion 58, 014034.

Osborne, T. H., Ferron, J. R., Groebner, R. J., Lao, L. L., Leonard, A. W., Mahdavi, M. A., Maingi, R., Miller, R. L., Turnbull, A. D., Wade, M., \& Watkins, J., 2000 The effect of plasma shape on H-mode pedestal characteristics on DIII-D. Plasma Phys. Control. Fusion 42, A175-A184.

Righi, E., Bartlett, D. V., Christiansen, J. P., Conway, G. D., Cordey, J. G., Eriksson, L.-G., De Esch, H. P. L., Fishrool, G. M., Gowers, C. W., DE HAAs, J. C. M., et al. 1999 Isotope scaling of the H mode power threshold on JET. Nucl. Fusion 39 (3), 309-319.

Rodriguez-Fernandez, P., Howard, N. T., Greenwald, M. J., Creely, A. J., Hughes, J. W., Wright, J. C., Holland, C., Lin Y., Sciortino, F. \& the SPARC Team 2020 Predictions of core plasma performance for the SPARC tokamak. J. Plasma Phys. 86. doi:10.1017/S0022377820001075.

Romanelli, F., KAMENDJE, R. on behalf of JET-EFDA Contributors 2009 Overview of JET results. Nucl. Fusion 49, 104006.

Ryter, F., Barrera Orte, L., Kurzan, B., McDermott, R. M., Tardini, G., Viezzer, E., Bernert, M., Fischer, R. \& the ASDEX Upgrade TeAm 2014 Experimental evidence for the key role of the ion heat channel in the physics of the L-H transition. Nucl. Fusion 54, 083003 . 
Ryter, F., Fuchs, J. C., Schneider, W., Sips, A. C. C., Stäbler, A., Stober, J. \& ASdeX UPGRADE TEAM $2008 \mathrm{H}$-mode confinement properties close to the power threshold in ASDEX upgrade. J. Phys. Conf. Ser. 123, 012035.

Ryter, F. \& H Mode Database Working Group 1996 H Mode power threshold database for ITER. Nucl. Fusion 36 (9), 1217-1264.

Ryter, F., PÜtterich, T., Reich, M., Scarabosio, A., Wolfrum, E., Fischer, R., Gemisic Adamov, M., Hicks, N., Kurzan, B., Maggi, C., et al. 2009 H-mode threshold and confinement in helium and deuterium in ASDEX upgrade. Nucl. Fusion 49, 062003.

Ryter, F., Rathgeber, S. K., Barrera Orte, L., Bernert, M., Conway, G. D., Fischer, R., Happel, T., Kurzan, B., McDermott, R. M., Scarabosio, A., et al. 2013 Survey of the H-mode power threshold and transition physics studies in ASDEX upgrade. Nucl. Fusion 53, 113003 .

Schmidtmayr, M., Hughes, J. W., Ryter, F., Wolfrum, E., Cao, N., Creely, A., Howard, N., Hubbard, A., Lin, Y., ReInKe, M., et al. 2018 Investigation of the critical edge ion heat flux for $\mathrm{L}-\mathrm{H}$ transitions in Alcator C-Mod and its dependence on $B_{\mathrm{T}}$. Nucl. Fusion 58, 056003.

Scott, S., Kramer, G. J., Snicker, A., Varje, J., Särkimäki, K., Tolman, E. A., RodrigueZ-FERnANDEZ, P. \& Wright, J. C. 2020 Fast ion physics in SPARC. J. Plasma Phys. 86. doi: $10.1017 / \mathrm{S} 0022377820001087$.

Silvagni, D., Eich, T., Faitsch, M., Happel, T., Sieglin, B., David, P., Nille, D., Gil, L., Stroth, U., the ASDEX Upgrade team \& the EUROfusion MST1 team. 2020 Scrape-off layer (SOL) power width scaling and correlation between SOL and pedestal gradients across L, I and H-mode plasmas at ASDEX Upgrade. Plasma Phys. Control. Fusion 62, 045015.

Snyder, P. B., Groebner, R. J., Hughes, J. W., Osborne, T. H., Beurskens, M., LeOnard, A. W., Wilson, H. R. \& XU, X. Q. 2011 A first-principles predictive model of the pedestal height and width: development, testing and ITER optimization with the EPED model. Nucl. Fusion 51, 103016.

Snyder, P. B., Wilson, H. R., Osborne, T. H. \& Leonard, A. W. 2004 Characterization of peeling-ballooning stability limits on the pedestal. Plasma Phys. Control. Fusion 46, A131-A141.

Snyder, P. B., Solomon, W. M., Burrell, K. H., Garofalo, A. M., Grierson, B. A., Groebner, R. J., Leonard, A. W., NAZikian, R., Osborne, T. H., Belli, E. A., et al. 2015 Super H-mode: theoretical prediction and initial observations of a new high performance regime for tokamak operation. Nucl. Fusion 55, 083026.

Snyder, P. B., Hughes, J. W., Osborne, T. H., Paz-Soldan, C., Solomon, W. M., Knolker, M., Eldon, D., Evans, T., Golfinopoulos, T., Grierson, B. A., et al. 2019 High fusion performance in Super H-mode experiments on Alcator C-Mod and DIII-D. Nucl. Fusion 59, 086017.

Solomon, W. M., Burrell, K. H., Fenstermacher, M. E., Garofalo, A. M., Grierson, B. A., Loarte, A., McKee, G. R., NAzikian, R., Osborne, T. H. \& Snyder, P. B. 2015 Extending the physics basis of quiescent H-mode toward ITER relevant parameters. Nucl. Fusion 55, 073031.

Stefanikova, E., Frassinetti, L., SaArelma, S., Loarte, A., Nunes, I., Garzotti, L., Lomas, P., Rimini, F., Drewelow, P., Kruezi, U., et al. 2018 Effect of the relative shift between the electron density and temperature pedestal position on the pedestal stability in JET-ILW and comparison with JET-C. Nucl. Fusion 58, 056010.

Sun, Y., Liang, Y., Liu, Y. Q., Gu, S., YAng, X., Guo, W., Shi, T., Jia, M., Wang, L., Lyu, B., et al. 2016 Nonlinear transition from mitigation to suppression of the edge localized mode with resonant magnetic perturbations in the EAST tokamak. Phys. Rev. Lett. 117, 115001.

Suttrop, W., Eich, T., Fuchs, J. C., Günter, S., Janzer, A., Herrmann, A., Kallenbach, A., LANG, P. T., Lunt, T., MARASCHEK, M., et al. 2011 First observation of edge localized modes mitigation with resonant and nonresonant magnetic perturbations in ASDEX upgrade. Phys. Rev. Lett. 106, 225004

TAKizuKa, T. \& ITPA H-Mode Power Threshold Database Working Group 2004 Roles of aspect ratio, absolute $B$ and effective $Z$ of the H-mode power threshold in tokamaks of the ITPA database. Plasma Phys. Control. Fusion 46, A227-A233. 
Tolman, E. A., Hughes, J. W., Wolfe, S. M., Wukitch, S. J., LaBombard, B., Hubbard, A. E., Marmar, E. S., Snyder, P. B. \& Schmidtmayr, M. 2018 Influence of high magnetic field on access to stationary H-modes and pedestal characteristics in Alcator C-Mod. Nucl. Fusion 58, 046004.

Walk, J. R., Snyder, P. B., Hughes, J. W., Terry, J. L., Hubbard, A. E. \& Phillips, P. E. 2012 Characterization of the pedestal in Alcator C-Mod ELMing H-modes and comparison with the EPED model. Nucl. Fusion 52, 063011.

Walk, J. R., Hughes, J. W., Hubbard, A. E., Terry, J. L., Whyte, D. G., White, A. E., Baek, S. G., Reinke, M. L., Theiler, C., Churchill, R. M., et al. 2014 Edge-localized mode avoidance and pedestal structure in I-mode plasmas. Phys. Plasmas 21, 056103.

Whyte, D. G., Hubbard, A. E., Hughes, J. W., Lipschultz, B., Rice, J. E., Marmar, E. S., Greenwald, M., Cziegler, I., Dominguez, A., Golfinopoulos, T., et al. 2010 I-mode: an $\mathrm{H}$-mode energy confinement regime with L-mode particle transport in Alcator C-Mod. Nucl. Fusion 50, 105005.

Wilks, T. M., Garofalo, A. M., Diamond, P. H., Guo, Z. B., Hughes, J. W., Burrell, K. H. \& CHEN, X. 2018 Scaling trends of the critical $E \times B$ shear for edge harmonic oscillation onset in DIII-D quiescent H-mode plasmas. Nucl. Fusion 58, 112002.

Yan, Z., Gohil, P., McKee, G. R., Eldon, D., Grierson, B., Rhodes, T. \& Petty, C. C. 2017 Turbulence and sheared flow structures behind the isotopic dependence of the $\mathrm{L}-\mathrm{H}$ power threshold on DIII-D. Nucl. Fusion 57, 126015. 